\title{
Reconfigurable Teams: Cooperative Goal Seeking with Self-Reconfigurable Robots
}

\author{
Zack Butler and Eric Fabricant \\ Department of Computer Science \\ Rochester Institute of Technology \\ Rochester NY USA \\ $\{z j b$, erf9559\}@cs.rit.edu
}

\begin{abstract}
Summary. Self-reconfigurable (SR) robots have been developed with the promise of enabling a wide range of abilities within a single hardware system. Particularly in the context of locomotion, SR robots may be able to traverse a wider range of environments than traditional legged or wheeled robots. Additionally, with a large number of modules, the system may be able to divide to explore areas in parallel and recombine to make larger, more capable groups. However, the choice of how to divide and merge these "reconfigurable teams" to most effectively solve tasks such as exploration of unknown terrain remains unanswered. This exploration problem can be seen as a superset of traditional multi-robot exploration, as robots must not only choose places to visit but also may coordinate to dynamically adjust the number of entities in the team. In this paper, we present a state-based distributed control algorithm for entities within a reconfigurable team that uses local sensory information and a team model to choose actions within the environment. We show a set of empirical results from simulation that show how the best choice of strategy may depend on the nature of the environment.
\end{abstract}

\section{Introduction}

Self-reconfigurable (SR) robots are systems composed of a group of modules which can change their relative topology to achieve different tasks. For example, a group of modules can construct a tower to position a sensor high above the ground, but then reform into a long narrow snake shape to maneuver through a narrow opening in a wall. In this work, we are interested in the idea that a system with a large number of modules may be able to form several independent groups, and work as a team of mobile robots, but with new and interesting capabilities. We introduce the term reconfigurable teams to suggest that not only is the overall system composed of self-reconfiguring modules which can make different shapes as the situation warrants, but that the entire team of robots can be reconfigured to have more or fewer members with variable size and capability during task execution. This can allow 
tasks such as exploration to be completed in parallel when possible while still retaining the ability to form larger, more capable robots as necessary.

Previous work in SR robotics has generally proceeded from the bottom up. Several different hardware systems have been developed, followed by centralized reconfiguration control and distributed control for both locomotion and reconfiguration. The first proposal was CEBOT [5], which included fairly complex physically cooperative robot modules, but following systems concentrated on simpler homogeneous modules, both lattice-based (in which modules fill space densely) [15,9] and chain-based (in which links of modules can move effectively without reconfiguration) $[16,3]$. More recent hardware such as MTRAN [10] and Superbot [13] can take on the characteristics of either type. Working from a biological viewpoint, the Swarm-Bots project [6] has developed a mobile robot design capable of self-assembly and disassembly for physical tasks such as hole-crossing, while retaining completely independent motion by each robot module, unlike most SR systems. We note that although the algorithms presented here are inspired by our work with latticebased robots, they are designed to be general enough to be applied to any of these systems.

Control algorithms for SR systems have primarily focused on two problems: reconfiguration, or how to generate a particular desired shape or topology of modules; and locomotion, or how to move a group of modules across their environment. Some early hardware-specific reconfiguration algorithms were proposed by Vona and Rus [12] and Yim et al. [17]. Locomotion for chain-based systems has been described by Zhang et al. [18] and Shen et al [14]. More generic algorithms for locomotion of lattice-based systems have also been described [4]. We have also previously described ways in which a group of lattice-based modules can split into two components and recombine with no centralized control in the context of locomotion [2]. Our work here implicitly assumes that these problems have been solved for the particular hardware system being used. That is, we look a group of SR modules as a single mobile entity which can navigate across its environment (within given physical constraints) and split and merge when desirable, and we are developing techniques to leverage these capabilities.

This work is also quite similar to multi-robot exploration, in which a team tries to visit and map an unknown environment under distributed control. Previous work in this area tends to concentrate on maximizing the amount of map area discovered rather than explicit goal locations, and tends to use more fine-grained cooperation than our technique $[1,7]$. Our technique also does not create a full map as in most previous work, but instead relies on local sensing of the environment. It is also partially inspired by the ALLIANCE architecture for general multi-robot task allocation [11], in which robots have different tasks to perform, but can become impatient and either give up their task or move to assist another robot that is not completing its job. We similarly use a state-based architecture and have robots timeout if they cannot complete their task, though we also use explicit calls for help when a robot becomes 
stuck. Finally, biologically-inspired approaches to multi-robot foraging and exploration tasks have also been proposed (e.g. [8]). Our work is similar in its reactive nature, but we use more explicit messaging between entities than most of these techniques (for example, to claim a particular goal and direct others elsewhere rather than cooperation based on pheromones or implicit cooperation via appropriate control laws). The ability to split and recombine to change the structure of the system also requires a more complex decisionmaking process.

\section{Problem Description}

In this work, we are developing distributed algorithms for exploration in the context of reconfigurable teams. Rather than focusing on the motions of individual modules, which can vary significantly from one hardware implementation to another, we assume that the system consists of groups of modules with inherent locomotion capability. We use the term blob to denote such a group of modules acting as a member of a reconfigurable team, and blob reconfigurations to mean those actions in which either a blob splits into two smaller blobs or two smaller blobs merge into one larger one. Traditional reconfigurations will likely also occur within a blob to perform locomotion or other tasks, however these actions are not explicitly considered in this work.

As a domain focus, we are investigating the problem of exploration of an unknown environment with a team of self-reconfiguring robots. In particular, we consider the situation of an initially unknown environment with a set of predefined locations (goals) that need to be visited. This implies that the blobs share a global coordinate system that they can maintain their location within. More general exploration can be implemented simply by placing goal locations densely in the environment. We implement here only the coordination at the blob level, not at the individual module level, though coordination at that lower level is also important. Our goal is to develop distributed algorithms under which each blob can decide what goal to attempt to reach, as well as whether or not it should split (to achieve two goals in parallel) or merge with another blob (to form a blob with greater capability).

There is a spectrum of ways to approach this problem: with reactive control, with search-based planning, or with some intermediate approach blending the two. In a reactive approach, each blob chooses its action based on its immediately available information, which may include both sensor data and information about the states of other blobs. The planning approach involves searching all possible action sequences over a map (including both motion as well as splits and merges) to come up with optimal choices for future actions. Our work is toward the reactive end of this spectrum, where decisions are made based on current local information with only some localized path planning. This allows for much simpler computations and lower resource requirements 
on the modules, as detailed maps do not have to be maintained and searched, though potentially at the expense of completeness in the exploration process.

In the course of this work, we have abstracted the physicality of the SR system to focus more clearly on the problem of deciding how to apportion resources to the exploration tasks at hand. However, the specific allocation depends critically on the ability of the hardware. Thus, we define a set of system parameters that describe these capabilities. In particular, we define a maximum slope value $s l_{\max }$, such that any group is assumed to be able to climb any slope no steeper than that, and a step height $s t_{\max }$ as the height (measured in module diameters) that a blob can surmount regardless of slope. The idea here is that a lattice-based robot can form a tower to climb a relatively short cliff, while a chain-based robot may also be able to raise some of its modules in a self-supported structure. We also define a sensing radius $r_{s}$ within which the blob can detect the topography of its environment. For any of these parameters, we allow that they may depend explicitly on $q$, the number of modules in the blob (we also use $Q$ to denote the total number of modules in the system). They may also be constant - for example, chain-based systems that use cantilevered modules would have $s t_{\max }$ limited by motor strength rather than $q$. Specifically, our experiments use values inspired by our previous work in simulations of lattice-based systems: $s l_{\max }=45^{\circ}, s t_{\max }=q / 3$, and $r_{s}=3 q_{r}$, where $q_{r}=\sqrt[3]{2 q / 3 \pi}$ is the radius of a hemisphere with volume $q$. Of course any values can be used for these parameters depending on the intention of the simulation. Finally, we assume that all blobs can communicate with each other throughout the environment through wireless means.

\section{Algorithm Design}

As mentioned previously, we have chosen a primarily reactive distributed algorithm to control the blobs within our system. Our algorithm bases its decisions on the blob's sensor data about its immediate neighborhood along with one internal state and the reported states of the other blobs in the system. The system architecture is divided into two components, primarily for clarity of exposition. These are the Navigation-Communication Unit (NCU), which is responsible for the overall decision making, and the Local Navigation Unit (LNU) which does short-range path planning (within the blob's sensor radius) at the request of the NCU. These components are assumed to run as a single instance within each blob, either in a leader module or collectively.

\subsection{Local Navigation Unit}

The LNU is provided with three inputs to determine a good local path for the robot. The first of these is the global coordinate of the intended destination $(d)$, which is provided by the NCU. The second and third inputs are $2 r_{s} \times 2 r_{s}$ matrices of discretized environmental measurements, centered at the blob's 


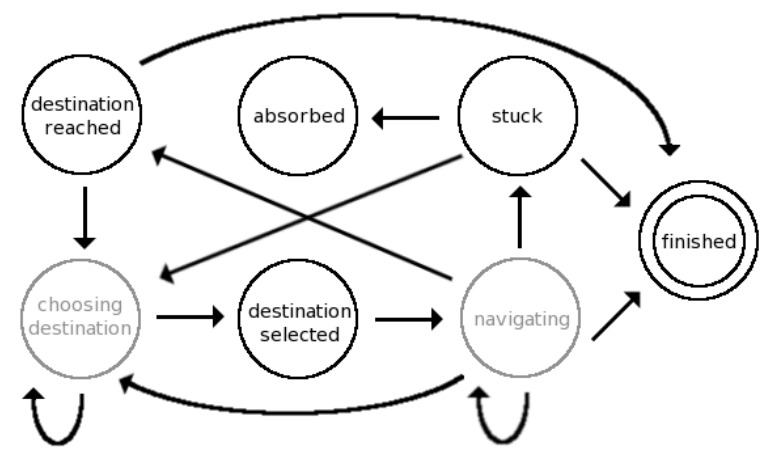

Fig. 1. State machine of the NCU. States outlined in black are communicated to the team, grey states are not.

position. Essentially, these matrices are grids that correspond to the section of the environment that a blob can perceive. One matrix consists of the local terrain's elevation values and the other their corresponding slopes. In our implementation of the LNU, square grids are used as a generalization of what a blob can sense to keep the sensory perimeter simple.

The purpose of the LNU is then to plan a navigable path to a point within the sensor radius that is as close as possible to $d$. A list of all points in the local grid are first sorted by favoring those closer to $d$. For each of these points, starting with the one closest to $d$, the shortest path from the blob's location to that point is computed and then tested for traversability according to the blob's physical limitations. That is, each sequence of points along the path with slope greater than $s l_{\max }$ constitutes a step, and so the height difference along that step is added together. If this total is greater than $s t_{\max }$, then this path is not navigable and the next possible local destination will be tested. If it is not (or there is no point along the path with a slope $>s l_{\max }$ ), then that local destination is returned to the NCU.

\subsection{Navigation Communication Unit}

The NCU operates by controlling a simple state machine that determines the overall behavior of the blob. It takes a list of goals as initial input and maintains a list of goal locations not yet reached by the team. It also monitors incoming messages from other blobs and maintains an active model of the team's state; when a blob signals the team of its state change, the remainder of the team use the notifier's identifier, status, and location to update the model. Specifically, the NCU keeps track of each blob's most recent intended destination, as well as which blobs cannot navigate in their desired direction due to the local topography. The list of goals and team model are used to produce its decision (and potentially an internal state change). 
As the NCU constantly references its model and sensor input, it may be in any of the seven internal states shown in Fig. 1. These are primarily in a loop of choosing a destination and informing the team, navigating toward it, and either reaching it or getting stuck. Along the way to a destination, it generally remains within the "navigating" state and does not reconsider its choice, though it may split itself along the way as described below. It uses the LNU to direct it toward the selected destination through intermediate points as long as possible. If at any point the LNU reports that there is no way to approach the desired destination, the blob transitions to the "stuck" state. It will then remain at its location as long as it knows that another blob is attempting to approach it. When no blob is actively en route to assist the stuck blob, the stuck blob will begin its timeout sequence. Upon timing out it will attempt to pursue a different destination to reduce the inefficiency of having its modules be inactive for prolonged periods of time.

When choosing a destination, the NCU is faced with decisions of whether to choose a goal location, split to attempt to achieve multiple goals, or combine with another blob to help the other blob to achieve its goal. In general, there is not necessarily a correct (or computably correct) choice amongst these possible actions, especially with a reactive algorithm. Without complete knowledge of the environment, it is not possible to say a priori whether it is faster to wait for another blob to surmount an obstacle or try a different goal and leave the closer goal for future exploration. With this in mind, we have developed and tested several different basic behaviors to see how the use of different heuristic choices impacts efficiency and reliability in different environments. In the "generous" behavior, the NCU is configured to give priority to blobs that are stuck over goal points, and will choose the closest such blob. Assisting blobs that are stuck increases the number of active modules on the team, improving the team's overall abilities by decreasing the likelihood of any given member becoming stuck. We allow multiple blobs to help a single immobile blob simultaneously, providing a degree of redundancy for unsticking in case one or more blobs are too small, whereas goal points are only pursued by one blob at a time because the blob can always request help if it needs it. We also tested a "greedy" behavior, in which the NCU will always choose the closest unclaimed goal location as long as one exists before helping any stuck blobs. This may be appropriate if the merge action is difficult, as the time taken to merge is not explicitly considered in our algorithm. Finally, we also tested a "neutral" behavior, in which the NCU chooses the closest potential destination, whether a stuck blob or a goal location, and a "limited" behavior in which splits and merges are not considered at all, to examine how much advantage is obtained from blob reconfigurations.

A blob may choose to split into two smaller blobs to improve parallelism throughout the mission. In order to ensure useful splitting, we define a lower bound on the group size $q_{l}$, such that each resulting blob must contain at least this many modules. This avoids producing blobs that are likely to get stuck, and since traversability depends on blob size, the value of $q_{l}$ should ideally 

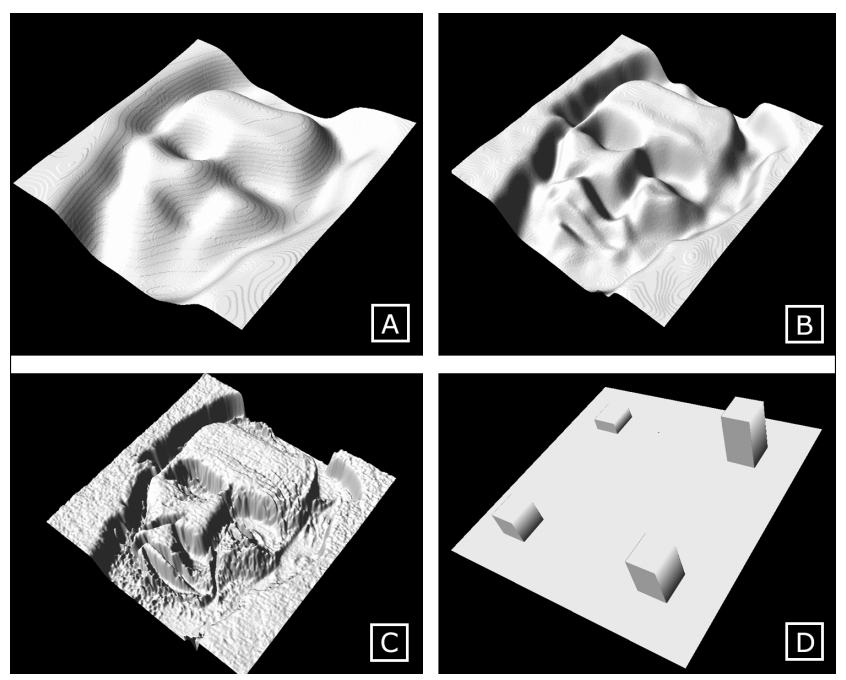

Fig. 2. Environments used for testing: (A)-(C) are increasingly more challenging versions of the same terrain, while (D) is constructed to require cooperation while allowing simple navigation through the majority of the environment. In our testing, goal locations were placed randomly for each run in environments (A)-(C) while in environment (D) one goal location was placed atop each pillar.

depend on the complexity of the environment, if known. In this work, we have chosen $q_{l}$ such that the smallest blob can surmount a step of $1 / 10$ the total elevation change of the environment. If this constraint can be satisfied, there are two times that a blob may split. First, it may split after reaching its destination and before choosing a new destination. Second, while navigating, if an alternate destination (i.e. an immobile blob or goal point) is within the blob's sensory radius, the LNU is capable of assessing what size blob is required to reach that point. If the total blob is sufficiently large, it will split such that one of the resulting blobs is just large enough to reach the visible destination. This approach conserves modules for the original destination while ensuring that the visible destination can be reached. In both cases, both blobs resulting from a split contain the same the full team model known prior to the split.

\section{Experimental Results}

In order to evaluate the utility of our algorithms, we performed a suite of experiments while varying different independent parameters, both environmental and algorithmic. The goal is to determine under what circumstances blob reconfigurations can be beneficial, either in terms of improved efficiency or more reliable goal attainment. We chose a set of environments, initial team sizes and configurations, and which of the algorithmic behaviors was used by 


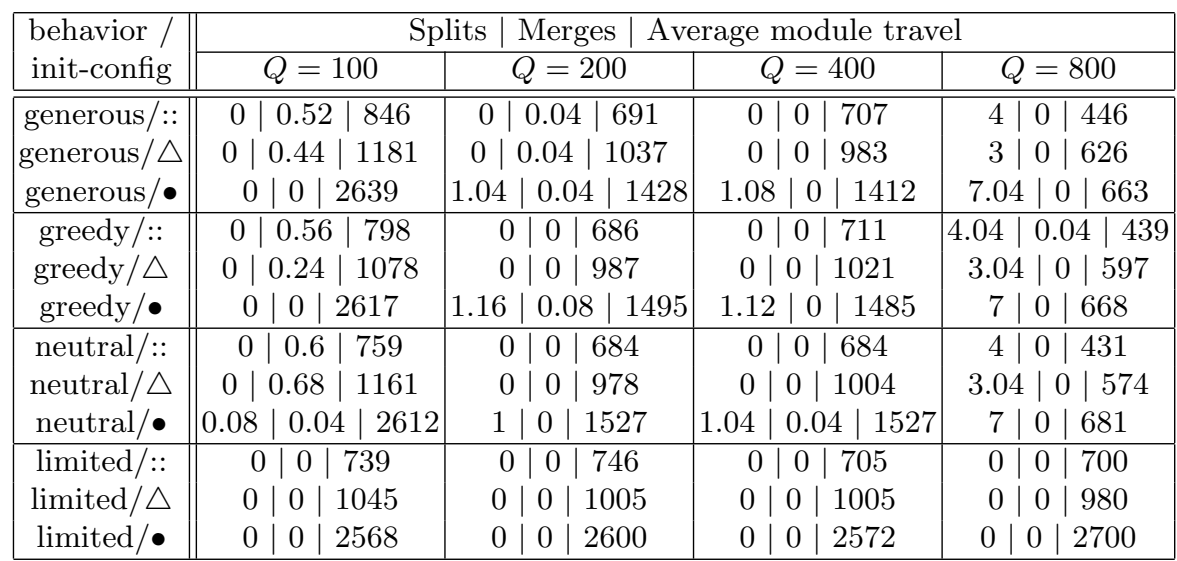

Table 1. Results from simpler environment (Fig. 2A) for different team sizes $Q$. Initial configurations are four groups near the corners of the environment (::), three groups in a triangle near the center $(\triangle)$ and one large group in the center $(\bullet)$. Each entry in the table represents the average of 25 trials.

the blobs, and tested all combinations of these parameters. For each set of parameters, we then measured the success rate (number of goals achieved) as well as the total travel distance and number of blob reconfigurations taken to achieve the goals. Total travel distance is measured as the average distance traveled by a module in the system, and for most systems indicates the total energy consumed during the mission. To compute this, the distance traveled by a blob during its lifetime is weighted by the size of the blob and the total over all blobs is divided by the team size $Q$.

To perform these experiments, we developed a graphical simulator for reconfigurable teams. The simulator spawns a separate thread for each blob to inject some of the non-determinism inherent in a real system, and uses an additional thread to handle communication between the blobs.

Four different environments were developed for testing, as displayed in Fig. 2. All the environments were $500 \times 500$ module diameters in size. Three of them (Fig. 2A-C) have the same general shape, with a total elevation change of 255 module diameters but with increasing jaggedness that calls for larger blobs. That is, the easiest map was smoothed such that most slopes were less than $s l_{\max }$, while the most difficult had steps requiring nearly all modules to surmount. In each of these maps, 25 trials were run for each set of parameters, with 30 goal points placed at random locations for each trial. A fourth environment (Fig. 2D) is primarily flat but with tall pillars at each corner that need to be explored, requiring 100, 200, 300 and 400 modules to climb. For this case we used hard-coded goal locations, with one goal at the center of each pillar. In each environment, we tested three distinct initial configurations for the modules: in four groups near the corners of the environment, in three 


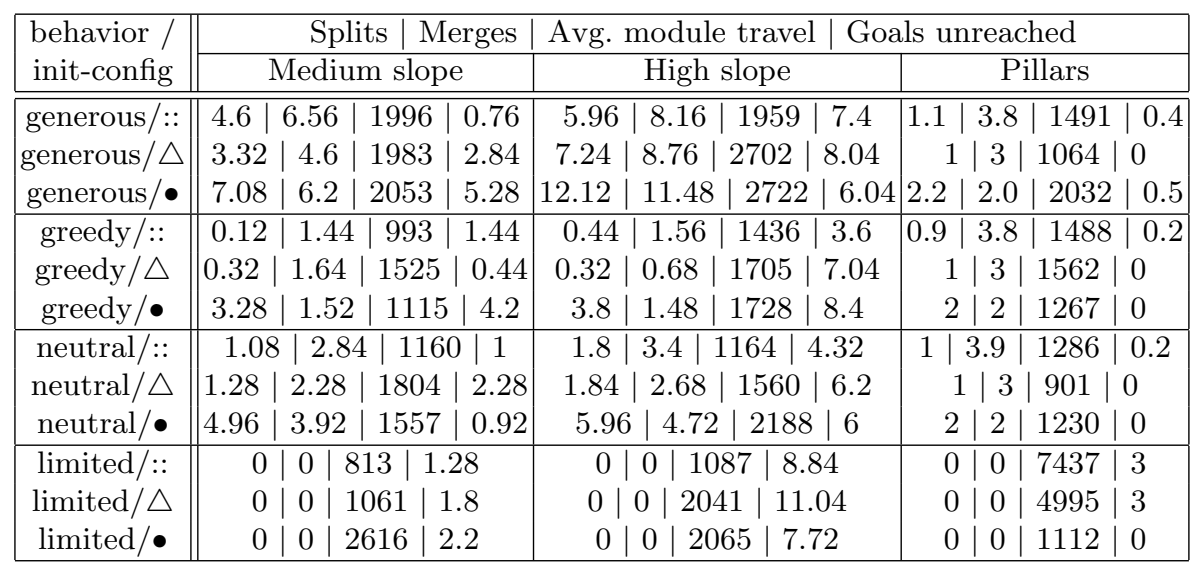

Table 2. Results from more challenging environments. Goals remaining are out of 30 initial goals for the first two environments and out of 4 for the Pillars environment.

groups in a triangle close to the center of the environment, and in one large group in the center.

On the easiest map (Fig. 2A), it was possible for the reactive technique to achieve all goal locations, and so we can compare the amount of work required under each robot size, initial configuration and behavior. The results are shown in tabular form in Table 1. In general, as expected, very few merge operations were required, since blobs were unlikely to get stuck, though some were used when the initial blob sizes were small (e.g. 100 modules divided among four groups). This has the effect that any of the self-reconfiguring behaviors will have substantially the same behavior, since there will be little need to be generous or greedy. On the other hand, with larger numbers of modules, splits did generally occur, helping the overall efficiency of the exploration. If the modules start in several groups, then the distance traveled is similar across the different behaviors, but if they start in one group (as would be a common deployment), then the use of splits dramatically decreases the distance that the modules have to travel.

On the more difficult terrains (Fig. 2B-C), due to the larger cliffs, we primarily tested with teams of 400 total modules. Here we can see how the different behaviors effect the reactive algorithm's ability to reach all desired locations. Perhaps surprisingly, the generous algorithm is in fact the least effective overall, using significantly more effort to achieve about the same or fewer goals. Our observations of these simulations are simply that the blobs spend too much effort attempting to help each other over the more challenging terrain and not enough going to reachable goals first. On the other hand, the greedy behavior does quite well, and in fact still shows a number of split and merge operations; it is simply that goal-directed behavior takes precedence and the reconfigurations allow for additional goals to be achieved in an implicit 
second phase. The neutral behavior generally takes more energy to reach a slightly larger number of goals than the greedy behavior, especially on the medium-difficulty environment (Fig. 2B). In the pillar environment (Fig. 2D), the reactive algorithm is put at somewhat of a disadvantage, since it will optimistically split before reaching the pillars and finding that it needs to merge again. When the initial group is large enough to climb all the pillars, the limited behavior is most efficient, but this behavior cannot reach three of the four goals unless it starts in a single large group. This environment seems to favor the neutral behavior that balances merges with goal locations to best form groups that can climb each pillar.

\section{Discussion / Future Work}

Overall, the use of blob reconfiguration in the context of reconfigurable teams has shown benefits in a variety of different situations. In particular, in environments that are more easily traversable, it allows any deployment to effectively explore in parallel, while in more challenging situations, the best behaviors can allow the system to collectively reach more goal locations more efficiently than without blob reconfigurations. The neutral behavior, in which destinations are chosen based purely on proximity rather than whether the destination represents a blob or a goal location, had the best overall success and efficiency, though a greedy behavior can be more efficient in difficult environments at the expense of achieving somewhat fewer goals.

One intriguing avenue of further study is the use of more complex mapbased algorithms instead of the simple reactive LNU used here. This should allow the blobs to reach more of the goals in the environment, but requires the modules to store and share large amounts of information as they explore. In the extreme case, with complete information, this becomes a superset of the multiple traveling salesmen problem (mTSP would define the "limited" behavior problem), which is NP-hard but has approximation algorithms available. However, we are still most concerned with cases where the initial environment is largely unknown. Using planning in a partially known environment will also require tighter integration of planning with the higher-level decision making to effectively choose long-term actions, since a blob will have to judge whether to take a potentially roundabout route to a goal or ask for help to make a larger blob that can take a more direct route.

Finally, we also plan to extend this work to handle heterogeneous SR systems. In such systems, modules have specialized capabilities, such as different sensors, and so blobs will be specified by their qualitative abilities as well as the quantitative parameters used in this work. Then, as in multi-robot task allocation, goals can be tagged (either a priori or after an initial detection) with the type of sensor that needs to visit that location, and the blobs will have to not only choose their goal location but also to split and merge with their sensor capabilities in mind. 


\section{References}

1. W. Burgard, M. Moors, D. Fox, R. Simmons, and S. Thrun. Collaborative multi-robot exploration. In IEEE International Conference on Robotics and Automation, pages 476-481, 2000.

2. Z. Butler, S. Murata, and D. Rus. Distributed replication algorithms for selfreconfiguring modular robots. In Distributed Autonomous Robotic Systems 5, pages $37-48,2002$.

3. A. Castano, W.-M. Shen, and P. Will. CONRO: Towards deployable robots with inter-robots metamorphic capabilities. Autonomous Robots, 8(3):309-24, 2000.

4. R. Fitch and Z. Butler. Million module march: Scalable locomotion for large selfreconfigurable robots. Int'l. Jnl. of Robotics Research, 27(3-4):331-343, 2008.

5. T. Fukuda and T. Ueyama. Cellular Robotics and Microrobotic Systems. World Scientific, 1994.

6. R. Groß, M. Bonani, F. Mondada, and M. Dorigo. Autonomous self-assembly in swarm-bots. IEEE Trans. on Robotics, 22(6):1115-30, 2006.

7. A. Howard and L. Kitchen. Cooperative localisation and mapping. In Proc. of the 1999 Int'l. Conf. on Field and Service Robotics, 1999.

8. C. R. Kube and H. Zhang. Collective robotic intelligence. In Proc. of the Second Int'l. Conf. on Simulation of Adaptive Behavior, pages 460-8, 1992.

9. S. Murata, H. Kurokawa, E. Yoshida, K. Tomita, and S. Kokaji. A 3-D selfreconfigurable structure. In Proc. of IEEE ICRA, pages 432-9, May 1998.

10. S. Murata, E. Yoshida, A. Kamimura, H. Kurokawa, K. Tomita, and S. Kokaji. M-TRAN: Self-reconfigurable modular robotic system. IEEE/ASME Trans. on Mechatronics, 7(4):431-41, 2002.

11. L. E. Parker. ALLIANCE: An architecture for fault tolerant, cooperative control of heterogeneous mobile robots. In Proc. of IEEE Int'l Conf. on Intelligent Robots and Systems, pages 776-83, Munich, Sept. 1994.

12. D. Rus and M. Vona. Self-reconfiguration planning with unit compressible modules. In Proc. of IEEE ICRA, pages 2513-20, 1999.

13. W.-M. Shen, M. Krivokon, H. Chiu, J. Everist, M. Rubenstein, and J. Venkatesh. Multimode locomotion for reconfigurable robots. Autonomous Robots, 20(2):165-177, 2006.

14. W.-M. Shen, B. Salemi, and P. Will. Hormone-inspired adaptive communication and distributed control for conro self-reconfigurable robots. IEEE Transactions on Robotics and Automation, October 2002.

15. J. Suh, S. Homans, and M. Yim. Telecubes: Mechanical design of a module for self-reconfigurable robotics. In Proc. of IEEE ICRA, pages 4095-4101, 2002.

16. M. Yim, D. Duff, and K. Roufas. PolyBot: a modular reconfigurable robot. In Proc. of IEEE ICRA, pages 514-20, 2000.

17. M. Yim, Y. Zhang, J. Lamping, and E. Mao. Distributed control for 3D metamorphosis. Autonomous Robots, 10(1):41-56, 2001.

18. Y. Zhang, M. Yim, C. Eldershaw, D. Duff, and K. Roufas. Scalable and reconfigurable configurations and locomotion gaits for chain-type modular reconfigurable robots. In Proceedings of the International Symposium on Computational Intelligence in Robotics and Automation, pages 893-9, 2003. 